\title{
Erratum to: a posteriori stabilized sixth-order finite volume scheme for one-dimensional steady-state hyperbolic equations
}

\author{
Stéphane Clain $^{1}$ - Raphaël Loubère ${ }^{2}$. \\ Gaspar J. Machado ${ }^{1}$
}

Published online: 7 October 2017

(C) Springer Science+Business Media, LLC 2017

\section{Erratum to: Adv Comput Math DOI 10.1007/s10444-017-9556-6}

During typesetting, Figs. 8 and 21 got corrupted and the images shown in the online published version are not correct. The original publication was updated.

$\triangle$ Gaspar J. Machado gjm@math.uminho.pt

Stéphane Clain

clain@math.uminho.pt

Raphaël Loubère

raphael.1oubere@u-bordeaux.fr

1 Centre of Mathematics, University of Minho, Campus of Azurém, 4800-058 Guimarães, Portugal

2 CNRS and Institut de Mathématiques de Bordeaux (IMB), Université de Bordeaux, Talence, France 